\title{
Establishment of European Regional Ionosphere Model Based on Spherical Harmonic Functions
}

\author{
Mingze Zhang* \\ School of Geomatics, Liaoning Technical University, Fuxin 123000, Liaoning, China
}

*Corresponding author: Mingze Zhang, 641375496@qq.com

\begin{abstract}
In order to study the temporal and spatial variation characteristics of the regional ionosphere and the modeling accuracy, the experiment is based on the spherical harmonic function model, using the GPS, Glonass, and Galileo dualfrequency observation data from the 305th-334th day of the European CORS network in 2019 to establish a global ionospheric model. By analyzing and evaluating the accuracy of the global ionospheric puncture points, VTEC, and comparing code products, the test results showed that the GPS system has the most dense puncture electricity distribution, the Glonass system is the second, and the Galileo system is the weakest. The values of ionospheric VTEC calculated by GPS, Glonass and Galileo are slightly different, but in terms of trends, they are the same as those of ESA, JPL and UPC. GPS data has the highest accuracy in global ionospheric modeling. GPS, Glonass and Galileo have the same trend, but Glonass data is unstable and fluctuates greatly.
\end{abstract}

Keywords: Global ionosphere; VTEC; Spherical harmonic function model

Publication date: November 2021; Online publication: November 30, 2021

\section{Introduction}

The composition of the near-Earth space environment includes the middle and upper atmosphere (troposphere, stratosphere), ionosphere, and magnetosphere. The middle and upper atmosphere is located $60 \mathrm{~km}$ from the ground to the upper layer of the earth. The ionosphere is an important part of the near-Earth space environment. The molecules and atoms in the atmosphere located between about $60-2000 \mathrm{~km}$ in the upper earth are ionized by the sun's ultraviolet rays, X-rays and high-energy particles, forming a plasma region, which is called the ionosphere ${ }^{[1]}$. The area from the top of the ionosphere to tens of thousands of kilometers above the top is the magnetosphere. The free electrons and ions in the ionosphere are one of the main sources of errors in electromagnetic wave propagation, which will cause delay errors in navigation and positioning. The ionospheric delay is generally about a few meters, but when the sunspot activity is strong, the ionospheric electron density will increase, and the ionospheric delay will increase, reaching 10 meters or even tens of meters ${ }^{[2]}$. Therefore, weakening and eliminating the impact of ionospheric delay on navigation and positioning has become an urgent problem in the field of global navigation satellite system (GNSS) ${ }^{[3]}$. Related scientific research on the ionosphere (for example, ionospheric storms, ionospheric scintillation, geomagnetic storms, and abnormal changes in earthquakes and tsunamis) also requires permanent and continuous monitoring of the state of the ionosphere. Therefore, how to best obtain a continuous accurate ionospheric model with high spatial and temporal resolution on a global scale is a popular direction for precise positioning and space weather applications ${ }^{[4]}$.

\section{Modeling method}

When modeling the regional ionosphere, the experiment adopted the fifteenth-order spherical harmonic 
function (SHF) model, because it has an excellent mathematical structure and can better reflect the temporal and spatial distribution of the total electron content. The expression of the VTEC spherical harmonic function model is:

$$
\operatorname{VTEC}(\varphi, \lambda)=\sum_{n=0}^{N} \sum_{m=0}^{n}\left(\tilde{A}_{n m} \cos (m \lambda)+\tilde{B}_{n m} \sin (m \lambda)\right) \cdot \tilde{P}_{n m}(\cos \varphi)
$$

In the above formula, VTEC is the electron content in the vertical direction, $\tilde{A}_{n m}$ and $\tilde{B}_{n m}$ is the

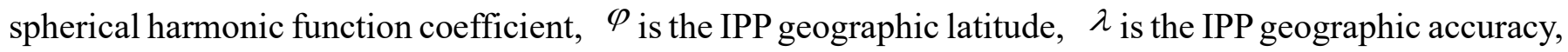
$\mathrm{n}$ is the degree of the spherical harmonic function, $\mathrm{m}$ is the order of the spherical harmonic function, $\tilde{P}_{n m}(\cos \varphi)$ is the normalized adjoint Legendre polynomial, Where $\mathrm{N}=15$-order spherical harmonic function model is used to describe the ionosphere.

\section{Data sources and experimental procedures}

This paper selects 240 CORS stations with a global uniform distribution for 30 days from the 305th day to the 334th day of 2019 as reference stations. The experiment uses GPS, Glonass, and Galileo observations to model the global ionosphere, and compares them with UPC, JPL, ESA and other institutions. The sampling interval of the observation data is 30 seconds, and the elevation mask angle of satellite is $10^{\circ}$. The height of the thin ionosphere is chosen to be $450 \mathrm{~km}$, the time resolution is $1 \mathrm{~h}$, and the spatial resolution is $5^{\circ} \times 2.5^{\circ}$. The broadcast ephemeris is provided by IGS. In this experiment, the European region ionosphere modeling adopts the fifteenth-order spherical harmonic function model, and the output is performed according to the standard format of ionospheric grid.

\section{Analysis of ionospheric pierce point}

When the electromagnetic wave source propagates from outer space to a certain point on the earth, the intersection of the electromagnetic beam entering the ionosphere is called the Ionospheric Pierce Point (IPP). This paper analyzes the characteristics of IPP and the accuracy of the ionospheric observable values of Global Positioning System, Glonass and Galileo. Figure 1 shows the IPP distribution of the three systems within 2 hours (UT00:00-02:00) on November 4, 2019. As shown in Figure 2, the IPP distribution of the GPS system is the densest, covering most of the continents of the world. Because the GPS system has 24 GPS satellite constellations with global coverage rate as high as $98 \%$, more than 4 satellites can be observed at any place and any time in the world, and the navigation information can be pre-stored in the satellites. GPS has a large number of tracking stations around the world, covering most parts of the world. With the completion of the Glonass restoration work, it has a good IPP distribution in most areas, but compared with the GPS system, although the puncture point distribution is roughly the same, the density is significantly lower than that of the GPS system. One of the main reasons for this is that there are currently only 13 satellites in operation, 5 of which are geostationary satellites, which cannot improve the spatial resolution of IPP distribution over time. In addition, the monitoring stations for tracking Beidou satellites are relatively limited and unevenly distributed, resulting in the number of IPP being much smaller than GPS. Due to the limitation of the number of satellites, the Galileo system has fewer independent positioning satellites than the Beidou system on a global scale. There is no doubt that with the increasing in the number of Beidou and Galileo satellites and the upgrading of the IGS tracking network, there will be more and more MGEX stations and Beidou and Galileo satellites in orbit; this means that the distribution of IPP will become very dense in most parts of the world. In addition, ionospheric monitoring capabilities will also be further strengthened. 


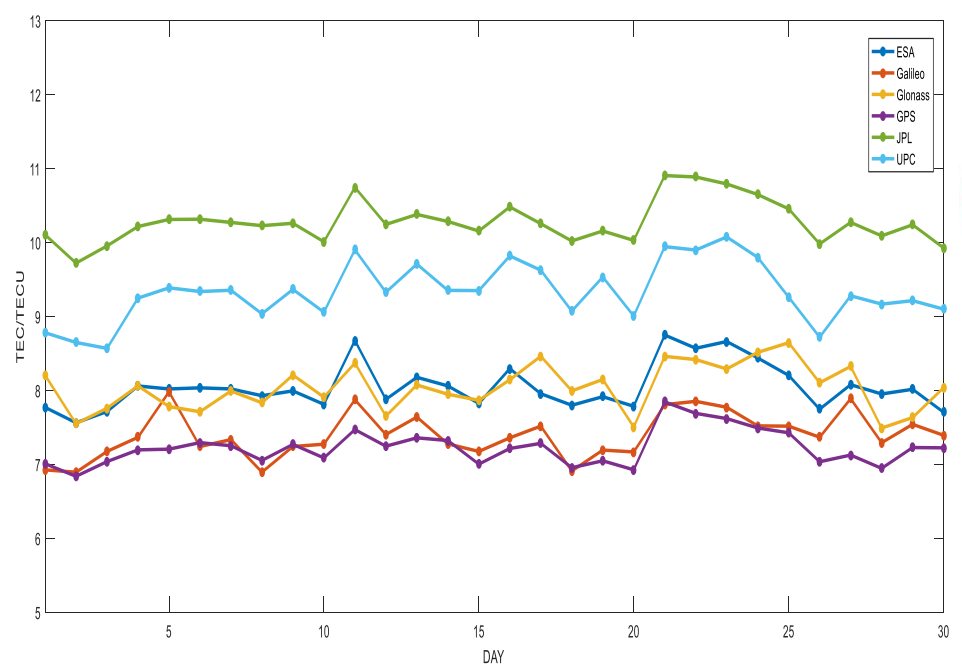

Figure 1. Daily average of global TEC

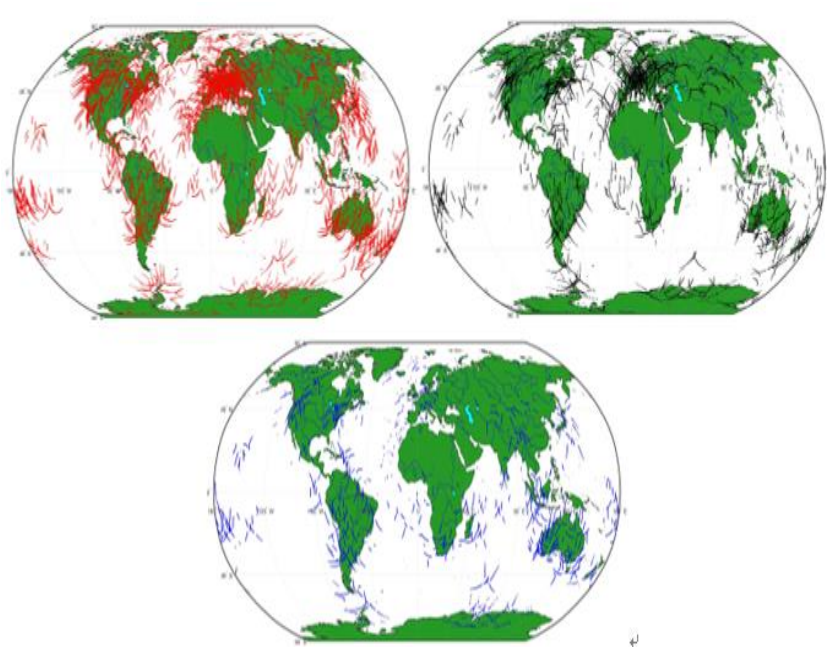

Figure 2. Distribution of pierce point

\section{VTEC accuracy analysis}

Figure 2. shows the daily average values of GPS, Glonass, Galileo, ESA, UPC and JPL global VTEC from the 305th to the 334th day of 2019, and the actual values for 30 days are shown in Table 1. Table 2 shows the correlation coefficient between the daily average of TEC of the three different systems and the daily average of TEC of ESA, UPC, and JPL.

Table 1. Detailed VTEC values of multi-system and multi-agency

\begin{tabular}{cccccccccccccc}
\hline Time & G & R & E & ESA & JPL & UPC & Time & G & R & E & ESA & JPL & UPC \\
\hline 1 & 7.01 & 8.21 & 6.93 & 7.77 & 10.11 & 8.78 & 16 & 7.22 & 8.14 & 7.36 & 8.29 & 10.48 & 9.82 \\
2 & 6.84 & 7.55 & 6.89 & 7.56 & 9.72 & 8.65 & 17 & 7.28 & 8.46 & 7.52 & 7.96 & 10.26 & 9.63 \\
3 & 7.04 & 7.76 & 7.18 & 7.71 & 9.95 & 8.57 & 18 & 6.96 & 7.99 & 6.91 & 7.80 & 10.02 & 9.07 \\
4 & 7.20 & 8.06 & 7.37 & 8.06 & 10.22 & 9.25 & 19 & 7.05 & 8.15 & 7.19 & 7.92 & 10.16 & 9.53 \\
5 & 7.21 & 7.78 & 7.98 & 8.02 & 10.31 & 9.39 & 20 & 6.93 & 7.51 & 7.17 & 7.79 & 10.03 & 9.01 \\
6 & 7.29 & 7.71 & 7.24 & 8.04 & 10.32 & 9.34 & 21 & 7.85 & 8.46 & 7.81 & 8.75 & 10.91 & 9.95 \\
7 & 7.25 & 7.99 & 7.33 & 8.02 & 10.27 & 9.36 & 22 & 7.69 & 8.42 & 7.85 & 8.57 & 10.89 & 9.90 \\
8 & 7.05 & 7.83 & 6.89 & 7.93 & 10.23 & 9.04 & 23 & 7.62 & 8.29 & 7.77 & 8.66 & 10.79 & 10.08 \\
9 & 7.27 & 8.21 & 7.24 & 7.99 & 10.26 & 9.37 & 24 & 7.49 & 8.51 & 7.52 & 8.44 & 10.65 & 9.80 \\
10 & 7.09 & 7.91 & 7.27 & 7.81 & 10.01 & 9.06 & 25 & 7.43 & 8.65 & 7.52 & 8.20 & 10.46 & 9.26 \\
11 & 7.47 & 8.37 & 7.87 & 8.68 & 10.75 & 9.90 & 26 & 7.04 & 8.10 & 7.37 & 7.75 & 9.98 & 8.72 \\
12 & 7.25 & 7.65 & 7.40 & 7.88 & 10.25 & 9.33 & 27 & 7.13 & 8.33 & 7.89 & 8.08 & 10.27 & 9.28 \\
13 & 7.36 & 8.07 & 7.64 & 8.18 & 10.38 & 9.71 & 28 & 6.95 & 7.49 & 7.29 & 7.95 & 10.09 & 9.17 \\
14 & 7.32 & 7.95 & 7.28 & 8.06 & 10.29 & 9.35 & 29 & 7.23 & 7.63 & 7.54 & 8.02 & 10.24 & 9.21 \\
15 & 7.00 & 7.87 & 7.17 & 7.82 & 10.16 & 9.35 & 30 & 7.22 & 8.04 & 7.39 & 7.70 & 9.93 & 9.10 \\
\hline
\end{tabular}

Table 2. Correlation coefficient table

\begin{tabular}{cccc}
\hline Correlation coefficient & ESA & JPL & UPC \\
\hline GPS & 0.94 & 0.91 & 0.94 \\
GLONASS & 0.72 & 0.74 & 0.73 \\
GALILEO & 0.72 & 0.71 & 0.77 \\
\hline
\end{tabular}


From Figure 2 and Table 1, it can be seen that GPS-GIM, Glonass-GIM and Galileo-GIM have the closest global TEC daily average values, but there are still differences between them. The possible reason is that the number of satellites observed by different systems is different, and in different systems the number of pierce points and the quality of the observed data are also different, which leads to differences in the average value. JPL-GIM has the largest global daily average values of TEC, which is about 3 TECU larger than GPS-GIM, because the modeling method used in this experiment is also different from the JPLGIM modeling method. The former uses a 15x15-order spherical harmonic function model; the latter uses a spherical bicubic spline model. The reason for the large difference between UPC-GIM and GPS-GIM is that UPC-GIM uses a spherical double-layer uniform grid model. The variation trend of GIM global TEC daily average values of different analysis centers tends to be the same, and the mutual difference is about $0 \sim 3.0$ TECU. ESA-GIM and GPS-GIM are similar in value, because they both use $15 \times 15$-order spherical harmonic function models to model the global ionosphere.

It can be seen from Table 2 that the generated GPS-GIM, Glonass-GIM and Galileo-GIM are correlated with the global TEC daily average values of different analysis centers. Among them, the correlation between GPS-GIM and other analysis centers is extremely strong and all are greater than 0.91. The correlations between Glonass-GIM and Galileo-GIM's other analysis centers are very strong, which are all greater than 0.7 . These prove that there is a correlation between different ionospheric modeling, but the correlation is weaker than that of GPS-GIM. The result is consistent with the conclusion of Figure 2.

\section{Conclusion}

This paper uses the GPS dual-frequency observation data of the European CORS station from the 326th day to the 332th day in 2019 to establish a global ionosphere model, and analyzes the reliability and stability of the established global ionosphere model, and draws the following conclusions.

(1) The IPP of the GPS system is the most densely distributed, covering most of the world's land. Compared with the GPS system, Glonass has roughly the same distribution of pierce point, but the density is significantly lower than that of the GPS system. The Galileo system has the smallest distribution of pierce point.

(2) Although the values of the ionospheric VTEC calculated by GPS, Glonass, and Galileo are slightly deviated, they have the same trend in terms of trends.

(3) The ionospheric products of multiple systems and various institutions have a strong correlation.

\section{Funding}

Key Research and Development Program of Liaoning Province (2020JH2/10100044); National Natural Science Foundation of China (41904037); National Key Basic Research and Development Program (973 Program) (2016YFC0803102)

\section{Disclosure statement}

The author declares no conflict of interest.

\section{References}

[1] Xie Y, Wu J, Chen J, et al., 2014, Global Ionospheric TEC Modeling Using Measured GPS and GLONASS. Geomatics and Information Science of Wuhan University, 39(08): 930-934.

[2] Liu J, Wang Z, Wang H, et al., 2008, Modeling Regional Ionosphere Using GPS Measurements over China by Spherical Cap Harmonic Analysis Methodology. Geomatics and Information Science of 
Wuhan University, 2008(08): 792-795+814.

[3] Wang Z, Wang K, Li H, et al., 2018, Characteristics of the Antarctic Ionosphere by Utilizing Spherical Cap Harmonic Analysis. Science of Surveying and Mapping, 43(10): 33-38.

[4] Kou R, 2019, Regional Ionospheric TEC Modeling and Spatio-temporal Variation Characteristics Analysis. Lanzhou Jiaotong University. 